\title{
Equipamiento y metodología para la determinación de la vida de materiales para moldes
}

\author{
A. Abuin*, H. Galarraga*, I. Crespo*, L. M. Plaza*, P. Carnicer*, I. Vicario* y J.C. García*
}

\begin{abstract}
Resumen Los moldes empleados en la fundición inyectada de metales no férreos tienen una vida útil limitada, debido al deterioro del molde, que se manifiesta en forma de grietas superficiales. Dichas grietas son causadas, fundamentalmente, por el fenómeno de fatiga térmica, debido a los sucesivos ciclos de calentamiento-enfriamiento de la superficie del molde durante su trabajo. La exigencia de mejorar los materiales para alargar la vida de los moldes, hace necesario el desarrollo de nuevos equipamientos para evaluar su comportamiento durante los ciclos de trabajo. INASMETTECNALIA, en colaboración con la FEAF (Federación Española de Asociaciones de Fundidores), ha desarrollado una máquina para determinar el comportamiento de los aceros empleados para la fabricación de moldes frente al fenómeno de la fatiga térmica. La máquina de ensayos es capaz de ensayar cuarenta probetas simultáneamente, constituyendo una gran ventaja respecto a las máquinas desarrolladas anteriormente. Su versatilidad, en cuanto a los sistemas de calentamiento y enfriamiento, hace posible que se puedan ensayar todo tipo de materiales, en diferentes condiciones de fatiga térmica.
\end{abstract}

\section{Test equipment and methodology for die material service life evaluation}

\begin{abstract}
Dies used in the non ferrous die casting process, have a limited service life due to the damage effect by surface cracking appearance. These cracks are mainly produced by thermal fatigue, which are promoted by a repeated heating and cooling process through working cycles. The existing demand to improve materials to extend the die's service life leads to the development of new equipments to test the material behaviour. INASMET-TECNALIA in collaboration with FEAF (Spanish Federation of the Foundry Associations), has developed a testing machine to evaluate the thermal fatigue behaviour of steels used to manufacture dies. This testing machine is able to test forty samples simultaneously, getting a remarkable advantage in regard to previous developed test equipment. The versatility of heating and cooling system makes possible to test all kind of materials in very different thermal fatigue conditions.
\end{abstract}

Keywords

Thermal fatigue; Steel; Die; Die casting; Pressure casting.

\section{INTRODUCCIÓN}

La industria de la inyección a presión de metales ligeros emplea moldes cuyo coste incide en el $20 \%$ del coste total del proceso de fabricación de las piezas. La vida útil de los moldes se encuentra entre 60.000 y 400.000 inyectadas, en el caso de la fundición de aluminio. Esta vida se incrementa en los casos de inyección de magnesio y cinc. Sin embargo, baja drásticamente en el caso de las aleaciones de cobre.

El deterioro de los aceros de herramienta empleados en la fabricación del molde se debe a las complejas solicitaciones termo-mecánicas y físico-químicas que sufren éstos durante los ciclos de trabajo. Factores como la temperatura del metal, la presión de inyección y el choque térmico producido durante la inyección tienen una gran influencia en la vida útil del molde.

La principal causa de sustitución de los moldes de inyección es la aparición de grietas en zonas puntuales de la superficie del molde, las cuales se generan por fatiga térmica como resultante de los reiterados ciclos inyección-expulsión de la máquina de inyectar. Las grietas y roturas producidas en estos materiales son reparadas o, en el caso de de que sea necesario, se realiza el cambio de la parte del molde donde se ubica la huella de la pieza, con el fin de asegurar la calidad tanto superficial como estructural de las piezas inyectadas. Dichas reparaciones ocasionan paradas de producción, costes de reparación, etc., 
que provocan un aumento en los costes de fabricación de las piezas inyectadas.

Durante el ciclo de trabajo de una máquina de fundición inyectada de aluminio, la temperatura de la superficie del molde varía desde la temperatura en el momento de la inyección $\left(500^{\circ} \mathrm{C}\right)$ hasta la temperatura correspondiente a la etapa de lubrificación del molde para iniciar el nuevo ciclo $\left(150^{\circ} \mathrm{C}\right)($ Fig. 1).

En un principio, el fenómeno de fatiga térmica se produce en la capa superficial del molde donde el material alcanza el gradiente térmico más severo. A medida que aumenta el número de inyectadas realizadas en el molde, el fenómeno se extiende desde la superficie al interior del molde.

La diferencia de variaciones de temperatura, en función de la distancia respecto a la superficie del molde, produce tensiones internas debido a las repetidas contracciones/dilataciones producidas por el gradiente térmico sufrido por el material. Este fenómeno se exterioriza mediante la aparición de grietas superficiales, inicialmente de dimensiones microscópicas y, a medida que continua el molde en servicio, progresan a dimensiones macroscópicas, como se puede apreciar en la fotografía tomada a una probeta tras 100.000 ciclos de trabajo (Fig. 2).

La necesidad de alargar la vida útil de los moldes, mediante el desarrollo de materiales con mejores características, ha propiciado la creación de varios métodos de ensayo con el fin de poder evaluar la resistencia de un material frente al fenómeno de la fatiga térmica.

El ensayo más conocido es el test de inmersión que realiza CWRU (Case Western Reserve University), avalado por NADCA (North American Die Casting Association) y adoptado por Ford(C, para sus criterios

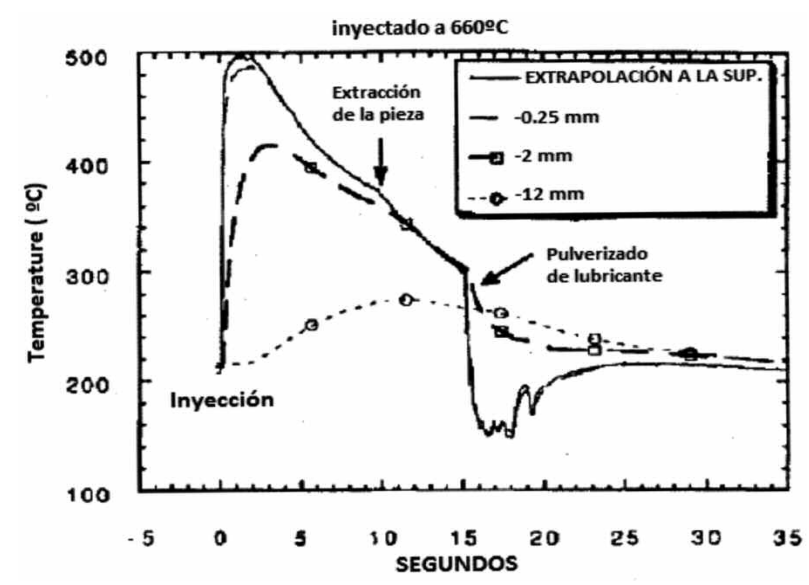

Figura 1. Variación de la temperatura con el tiempo a diferentes distancias de la superficie del molde durante el ciclo de trabajo ${ }^{[3]}$.

Figure 1. Temperature-time graph at different distances from the surface of the die ${ }^{[3]}$.

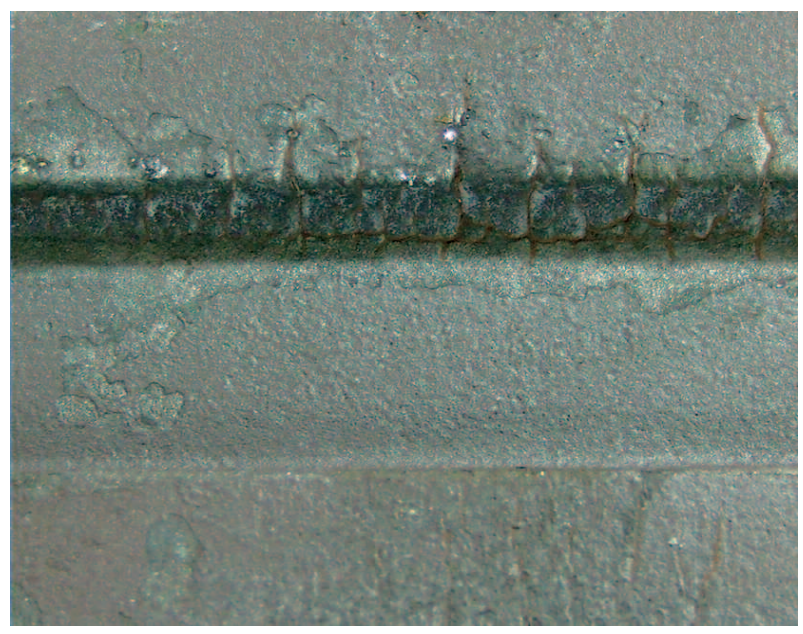

Figura 2. Grietas producidas por fatiga térmica en una probeta de acero. Figure 2. Thermal fatigue cracks in a steel
specimen.

de aceptación de aceros para moldes de inyección. Otros centros, como CTIF (Centro Tecnológico de la Fundición de Paris) y compañías como Uddeholm( $\mathrm{O}$ Böehler@ (fabricantes de aceros para herramientas) también han desarrollado sus propios ensayos de fatiga térmica para desarrollar materiales con mejores características frente a este fenómeno.

Todos los métodos de ensayos desarrollados hasta ahora, solo son capaces de ensayar un número reducido de materiales en cada batería de ensayos, lo que puede suponer una duración del ensayo de meses, como en el caso del CWRU (69 días para realizar un ensayo de 100.000 ciclos). Esto supone un gran inconveniente para el caso de realizar ensayos comparativos de aceros con diferentes composiciones, con distintos tratamientos térmicos, con diferentes recubrimientos, etc, ya que pueden durar desde varios meses a años.

Valorando las ventajas y limitaciones de los sistemas antes mencionados, se ha diseñado un nuevo sistema que permite conocer la resistencia a fatiga térmica de los materiales sobre probetas con geometrías variables.

Con esta nueva máquina de ensayos, se puede evaluar la influencia de todos los parámetros que entran en juego en el proceso de inyección tales como:

- El material: Se pueden comparar el comportamiento de aceros de distintas composiciones (calidades) o de distintos fabricantes.

- El tratamiento térmico del acero: Permite comparar el mismo acero con distintos tratamientos térmicos con el fin de optimizar el mismo con vistas a su comportamiento frente a la fatiga térmica. 
- El tratamiento superficial: Estudio comparativo de los distintos tratamientos superficiales existentes en el mercado para aumentar la resistencia a fatiga térmica del molde.

- La geometría: Se puede estudiar la influencia de distintos parámetros geométricos del molde frente a la fatiga térmica, como pueden ser los radios de curvatura, superficies de paredes delgadas, etc. De esta manera, se podría optimizar el diseño de las piezas inyectadas y, en consecuencia, de la huella del molde, con el objeto de tener geometrías del mismo con mejores propiedades frente a la fatiga térmica.

- La refrigeración: Evaluación frente a fatiga térmica de los diferentes métodos para la refrigeración exterior e interior del molde.

- La lubricación: Comparación de la resistencia de los moldes a la fatiga térmica en función de la lubricación empleada durante el ciclo de trabajo.

- Los parámetros básicos del proceso: Influencia de parámetros tales como: temperatura del metal y del molde, tiempos de ciclo, velocidades de inyección, presión de multiplicación, etc.

El nuevo sistema permite realizar estudios múltiples por la versatilidad de la máquina en cuanto al número de parámetros del proceso de inyección a ensayar en el equipo desarrollado. La complejidad de los parámetros del fenómeno exige la realización de ensayos de forma repetitiva para constatar la homogeneidad de los mismos, por lo que se hace necesaria la utilización de un equipo de ensayo capaz de ensayar varias probetas simultáneamente y con gran versatilidad en cuanto a la variación de sus parámetros.

\section{MÉTODO EXPERIMENTAL}

La máquina consta de un bastidor cúbico con una especie de carrusel que gira sobre un eje vertical ubicado en el centro del bastidor. Las probetas realizadas a partir de los materiales que se desean ensayar se cuelgan del "carrusel", de tal manera que puedan girar alrededor de un eje central. El eje central esta accionado por un motor-reductor y variador de velocidad con lo que se pueden lograr velocidades comprendidas entre 1 y $8 \mathrm{rpm}$.

En dos de los extremos de la máquina existen dispositivos de calentamiento y enfriamiento con lo que, por cada vuelta o ciclo del "carrusel", las probetas pasan por una zona de calentamiento y otra de enfriamiento (Fig. 3).

El sistema de calentamiento consiste en un inductor electromagnético de potencia regulable que genera

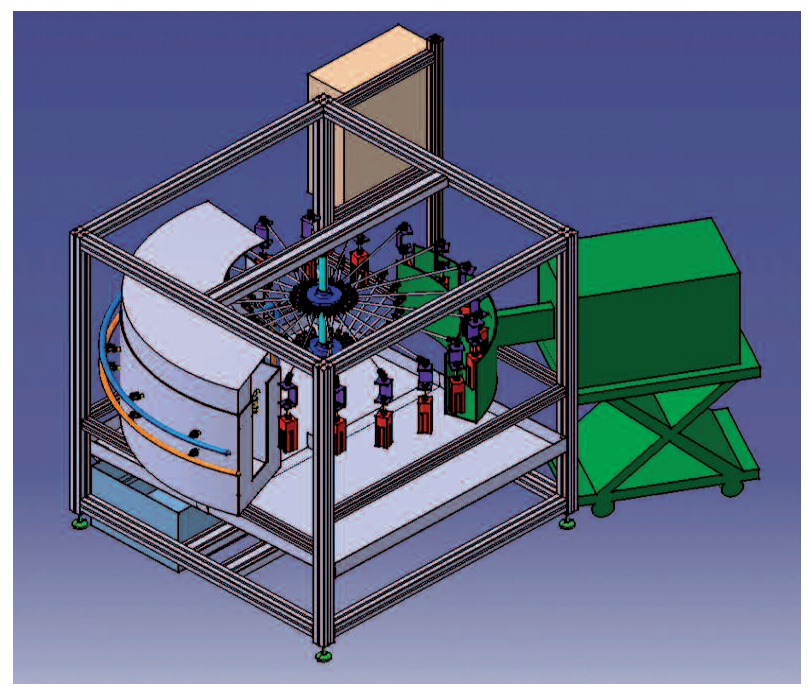

Figura 3. Esquema de la máquina de fatiga térmica con los sistemas de calentamiento y enfriamiento en los extremos.

\section{Figure 3. Thermal fatigue machine with heating and cooling systems at the corners.}

el calentamiento de la probeta mediante la aplicación de un campo magnético variable. El inductor está formado por una espira, a la cual se le ha dado una forma de dos placas concéntricas curvadas, por entre las que pasan las probetas.

El sistema de enfriamiento está compuesto por un recipiente de acero inoxidable donde se han colocado diez boquillas pulverizadoras aire-líquido, de tal manera que las probetas pasan por entre las boquillas. El circuito de refrigeración es un circuito cerrado, con dos depósitos de agua, uno, superior, donde se añade agua descalcificada compensando las pérdidas por evaporación y, otro, inferior, donde el agua recogida tras la pulverización se enfría.

Las temperaturas de trabajo se controlan mediante dos lectores de temperatura infrarrojos. El primero se ubica a la salida del inductor electromagnético, de tal manera que se pueda medir la temperatura máxima de la probeta. El segundo lector se ubica a la salida de la zona de enfriamiento, midiéndose la temperatura más baja del ciclo. Las temperaturas se registran en un ordenador, de modo que se puedan detectar variaciones del ciclo que pudieran alterar el resultado del ensayo (Fig. 4).

Las dimensiones principales de las probetas fabricadas para los ensayos que se han realizado, han sido tomadas de acuerdo a la probeta diseñada y empleada por el CWRU ${ }^{[4]}$. El resto de las dimensiones y formas han sido adoptadas con el fin de evaluar la influencia de la geometría sobre el comportamiento de los aceros frente a la fatiga térmica. Se han tenido 


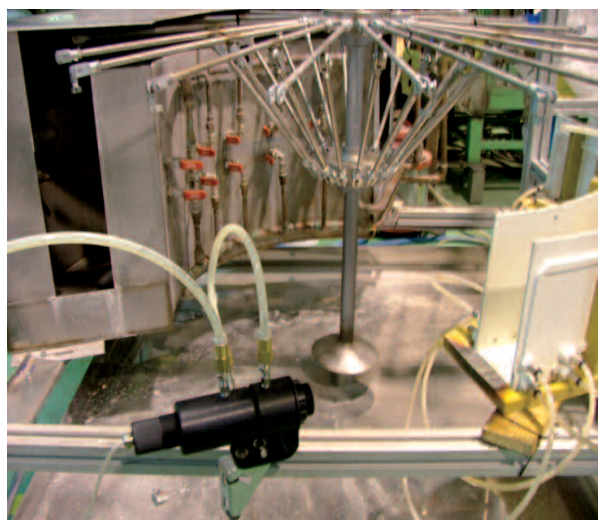

Figura 4. Máquina de fatiga térmica. En primer plano, se puede observar el lector de temperaturas a la salida del inductor.

Figure 4. Thermal fatigue machine. Temperature display at induction coil way out.

en cuenta parámetros, tales como los radios de curvatura de las aristas o los espesores de las paredes en la huella del molde (Fig. 5).

La máquina de fatiga térmica se ha diseñado de manera que tenga una gran flexibilidad, permitiendo así la realización de pruebas de muy variada índole. Los módulos de calentamiento y enfriamiento son intercambiables, dando la posibilidad, por ejemplo, de ubicar un sistema de calentamiento mediante gas en vez del actual, de inducción electromagnética. Incluso, es posible instalar un dispositivo que pueda elevar y bajar las probetas, logrando que éstas puedan sumergirse en aluminio fundido, mientras la máquina continúa girando. Es posible, también, el empleo de lubricador que permita simular la lubricación de los moldes de inyección en servicio, en lugar de agua como fluido refrigerante para las probetas, pudiéndose estudiar la influencia de las distintas composiciones y mezclas de refrigeración, en la vida útil de los aceros para moldes. La elevada versatilidad de la máquina, por lo tanto, permite realizar ensayos muy variados y ajustarlos a las necesidades del material a ensayar o incluso a las exigencias de los parámetros a evaluar. Con todo ello, se podría destinar la máquina a ensayos de fatiga térmica a materiales tan diferentes como: cerámicas, polímeros, hormigones, etc., además del acero y otros materiales metálicos.

El procedimiento para realizar el ensayo de fatiga térmica consiste en efectuar etapas de 10.000 ciclos hasta completar el ensayo fijado en 100.000 ciclos. Las velocidades de giro de las probetas que se han empleado hasta ahora han sido de 1 y $2 \mathrm{rpm}$ correspondientes a ciclos de 60 y $30 \mathrm{~s}$ de duración, respectivamente (Fig. 6).
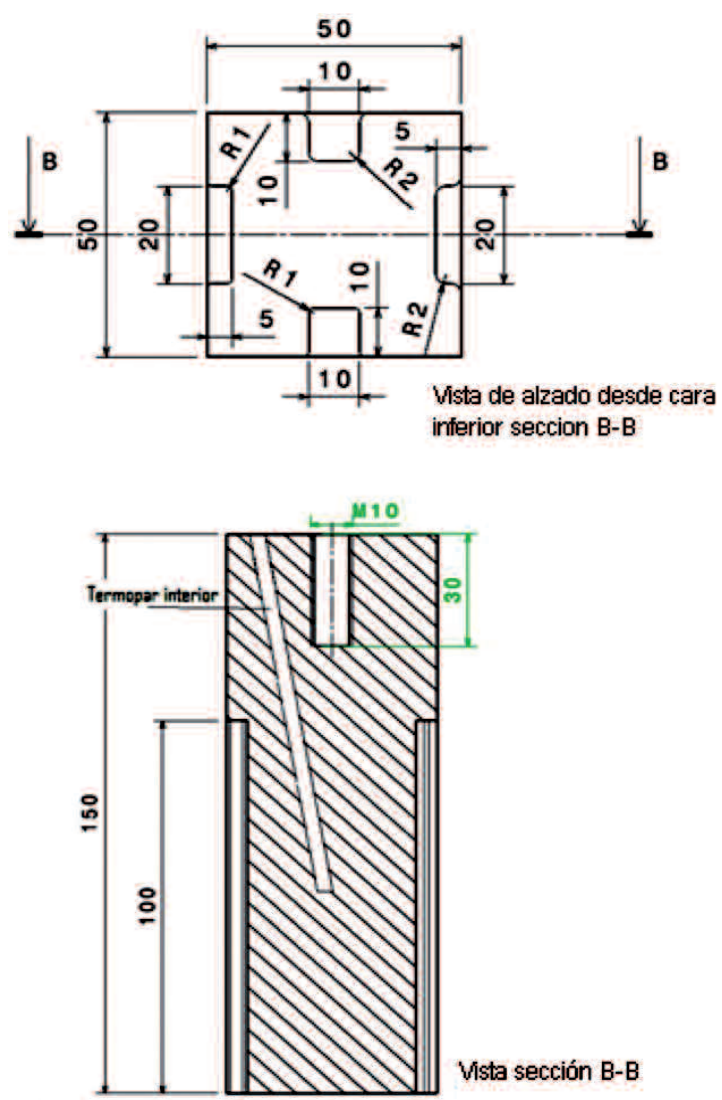

Figura 5. Plano constructivo de las probetas empleadas.

Figure 5. Constructive drawing of the specimens used.

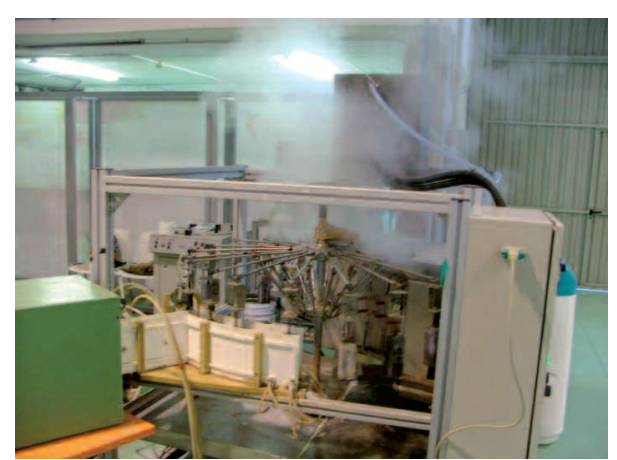

Figura 6. Máquina de fatiga térmica durante la realización de los ensayos.

Figure 6. Thermal fatigue machine doing a test.

\section{RESULTADOS Y DISCUSIÓN}

El ensayo se comienza con las probetas a temperatura ambiente. Durante los primeros ciclos, se realiza un calentamiento sin refrigeración y, una vez que la temperatura de las probetas se haya acercado a la 
temperatura de trabajo establecida, se acciona el enfriador para que las probetas realicen los ciclos de calentamiento-enfriamiento de modo continuo (Fig. 6). El rango de temperaturas en la superficie del material es de $650^{\circ} \mathrm{C}$ a la salida del calentamiento y $250^{\circ} \mathrm{C}$ a la salida del enfriamiento. La temperatura en el interior de la probeta tiene una variación de temperatura menor que en la superficie; las temperaturas máxima y minima medidas en el centro de la probeta son de $600^{\circ} \mathrm{C}$ y $500^{\circ} \mathrm{C}$, respectivamente. Dicha distribución de temperaturas es similar a la que tienen un macho o una zona de paredes finas en la huella del molde durante los ciclos de trabajo. Este factor se ha considerado como una ventaja a la hora de realizar el ensayo, debido a que los machos son las partes que más sufren por fatiga térmica en un molde de inyección.

Al final de cada etapa de 10.000 ciclos se evalúa el estado de la superficie de la probeta. Dicha evaluación consiste en un ensayo de partículas magnéticas, mediante la cual las grietas superficiales que hayan podido producirse debido a la fatiga térmica se hacen visibles a la luz ultravioleta. A continuación, se obtiene una fotografía, por cada cara de la probeta, para determinar el grado de deterioro de la misma. La cuantificación del deterioro se realiza mediante la comparación visual de las fotografías obtenidas con unos patrones de severidad (Fig. 7).

Una vez establecido el grado de severidad de cada una de las probetas ensayadas, se procedió a realizar el análisis comparativo de los resultados obtenidos. De esta manera, se pudo determinar la influencia de los distintos parámetros que afectan al deterioro del acero debido al fenómeno de la fatiga térmica (tipo de acero, tratamiento térmico, recubrimiento superficial, geometría, tipo de desmoldeante, etc.).

\section{CONCLUSIONES}

Se ha desarrollo un equipo de ensayos por fatiga térmica, capaz de realizar ensayos de fatiga térmica múltiples y de gran versatilidad en cuanto al calentamiento y enfriamiento. Incluso, se puede simular el efecto del lubricante utilizado para lubricar y enfriar el molde. Permite el estudio de la respuesta a la fatiga térmica de diferentes composiciones del material, con diferentes tratamientos térmicos y superficiales, diferentes medios de lubricación, geometría, tipo de desmoldeante, etc. La cuantificación del deterioro (grietas macroscópicas en su superficie) se realiza visualmente, con unos patrones de severidad desarrollados para los moldes de acero

El equipamiento ha sido validado mediante 60 pruebas realizadas sobre probetas de acero. Se han ensayado distintos aceros, con el fin de poder verificar la validez

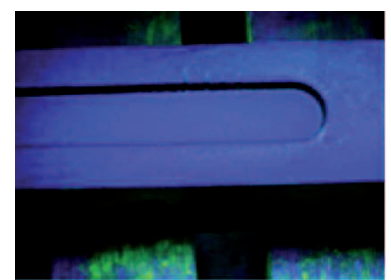

Patrón severiklad 1

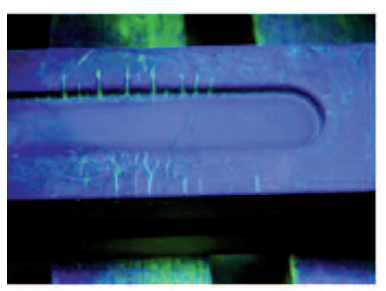

Patrón severidad 3

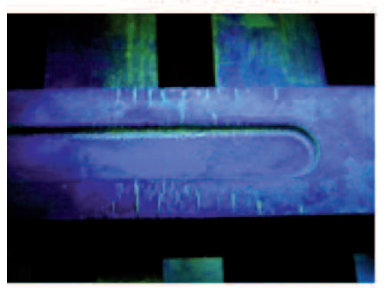

Patrón severidad 5

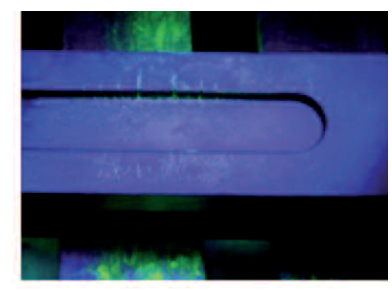

Patrón severílad 2

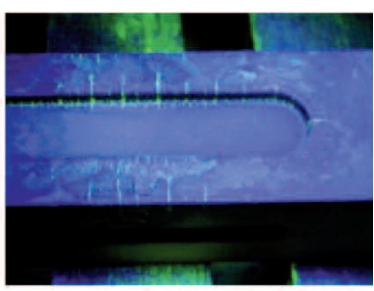

Patrón severidad 4

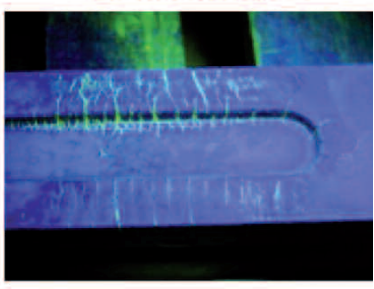

Patrón Severidad 6
Figura 7. Patrones de severidad empleados para evaluar el grado de deterioro de las probetas.

Figure 7. Severity patterns used to evaluate specimen deterioration degree.

de la máquina de ensayo sobre distintos materiales de partida.

El equipo de ensayo puede aplicarse, también, a estudios de fatiga térmica en otros tipos de materiales metálicos o no metálicos

\section{Agradecimientos}

Este trabajo ha sido patrocinado por la Comisión Técnica del Aluminio de la FEAF (Federación Española de Fundidores).

\section{REFERENCIAS}

[1] B. Kosec, Metalurgija 47 (2008) 51-55.

[2] D. Klobccar, J. Tusek y B. Taljat, Mater. Sci. Eng. 472 (2008) 198-207.

[3] J. Papai y C. Mobley, Trans. 16 ${ }^{\text {th }}$ Int. Die Casting Cong. Exp. NADCA, Detroit, Michigan, EE. UU., 1991, pp. 377-384.

[4] D. Schwan, J.F. Wallace y S. Birceanu, Final Technical Report, 2004. Department of Materials Science: Case Western Reserve University. Cleveland, Ohio, EE. UU., 2004. 